\title{
ASYMMETRIES OF ISOKINETIC STRENGTH AND FLEXIBILITY IN YOUNG SOCCER PLAYERS: A SYSTEMATIC REVIEW
}

review paper

() Wroclaw University of Health and Sport Sciences

DOI: https://doi.org/10.5114/hm.2022.108317

\section{SARAH RAMOS ${ }^{1,2 \oplus}$, MARCELO CORSO $^{1}$, AMANDA BROWN $^{1,3 \oplus}$, ROBERTO SIMÃO$^{1}$, INGRID DIAS ${ }^{1 \oplus}$}

${ }^{1}$ School of Physical Education and Sports, Federal University of Rio de Janeiro, Rio de Janeiro, Brazil

${ }^{2}$ Medical Department, Club de Regatas Vasco da Gama, Rio de Janeiro, Brazil

${ }^{3}$ Graduate Program in Clinical Medicine, Federal University of Rio de Janeiro, Rio de Janeiro, Brazil

\section{ABSTRACT}

Purpose. The study aim was to review the isokinetic strength assessment protocols, the obtained peak torque (PT) values, and the hamstring/quadriceps ratio $(\mathrm{H} / \mathrm{Q})$; review the unilateral evaluation protocols and normative values for flexibility; and verify the existence of asymmetries between the limbs, muscle imbalances, or bilateral differences (BD) in the strength and flexibility of young soccer players.

Methods. The PRISMA model was used. Searches were conducted in PubMed, SciELO, and LILACS, divided into soccer, isokinetic, and flexibility. Inclusion criteria: articles with a sample of young elite male soccer players with an average age less than 20 years; isokinetic strength assessment: presentation of values for absolute PT and/or H/Q ratio; flexibility assessment: use of unilateral tests for the hip flexion movement. The quality and risk of bias in each study were also analysed.

Results. In the isokinetic strength, a symmetrical pattern was observed for PT and the H/Q ratio between the limbs and divergent results for BD. Regarding flexibility, it was possible to verify a tendency towards an asymmetry of flexibility, with a more flexible dominant than non-dominant leg. Evaluation protocols and normative values were proposed for the tests of isokinetic strength and flexibility.

Conclusions. Imbalances for the H/Q ratio, asymmetries, and BD for isokinetic strength and flexibility are not natural aspects of the modality and should be avoided in young soccer players. The monitoring of these characteristics and the use of interventions to restore a symmetrical pattern have become essential since the beginning of sports life.

Key words: athletes, muscle strength dynamometer, muscle strength, range of motion, side-to-side differences

\section{Introduction}

Soccer is a complex sport that includes several physical valences, and among them, we can highlight the strength and flexibility. The International Federation of Association Football (FIFA, Fédération Internationale de Football Association) [1] states that the limb strength of soccer players is developed from specific activities of the modality, such as running, jumping, and kicking the ball, performed during the training and games period. Concerning flexibility, it is possible to verify that athletes who present a high degree of this valence consequently demonstrate a better proficiency in movements [2, 3].
It is worth mentioning that soccer players usually have a preferred leg to kick, defined as the dominant limb (DL), while the other serves as a support and is called the non-dominant limb (NDL). From this, it is possible to suggest that repeated actions, such as kicking, produce constant uneven workloads, creating asymmetries between DL and NDL [4]. This fact increases the chance of these athletes to develop injuries, especially when 2 or more asymmetric characteristics join [5].

As a way of measuring strength, possible asymmetries between DL and NDL, and imbalances in the hamstring/quadriceps (H/Q) relation, an isokinetic assessment can be used. It allows to obtain the values

Correspondence address: Sarah Ramos, Medical Department, Club de Regatas Vasco da Gama, Street General Almério de Moura 131, Vasco da Gama, 20921-060, Rio de Janeiro, Brazil, e-mail: sarah.ramos@outlook.com.br, https://orcid.org/0000-0002-3639-8343

Received: October 25, 2020

Accepted for publication: March 26, 2021

Citation: Ramos S, Corso M, Brown A, Simão R, Dias I. Asymmetries of isokinetic strength and flexibility in young soccer players: a systematic review. Hum Mov. 2022;23(4):21-33; doi: https://doi.org/10.5114/hm.2022.108317. 
of the absolute peak torque (PT), bilateral differences (BD) (difference between the PT of the quadriceps of DL and that of NDL, exposed in the form of a ratio or percentage values), and the $\mathrm{H} / \mathrm{Q}$ ratio [6]. On this theme, Costa Silva et al. [7] and de Lira et al. [8] did not find a significant number of young athletes who had $\mathrm{BD}$, considering values greater than $15 \%$ as representing asymmetry. The results achieved by Śliwowski et al. [9] and Maly et al. [10] differ from those previously mentioned; however, the authors used the value of $10 \%$ as the cut-off point. Some other studies verified asymmetry concerning PT, but did not examine it in relation to BD [11-13].

In the case of flexibility, it is necessary to measure this valence unilaterally to compare asymmetries between DL and NDL and possible BD. For this purpose, the importance of evaluating the hip flexion movement is highlighted, as it looks like the kicking movement and serves to assess the flexibility of the posterior chain of the lower limbs [3, 14]. Verifying this fact, Daneshjoo et al. [2] and Sanz et al. [15] observed that DL was significantly more flexible than NDL; in contrast, Akbulut and Agopyan [3] and Ramos et al. [16] found no difference between the limbs.

In addition to the possible influence of asymmetries, imbalances in the $\mathrm{H} / \mathrm{Q}$ ratio and $\mathrm{BD}$, young soccer players are in an incomplete maturation and growth phase, which generates a higher probability of injury [17]. Another point worth mentioning is that one of the main risk factors for triggering injuries is recurrence [18]. Therefore, through the evaluation of these factors, it is possible to detect and intervene to compensate for these disproportions at the beginning of the training life of these young players [9], acting in the injury prevention process.

From the above, one can observe that with reference to strength and flexibility in young soccer players, the literature still seems to be confused concerning possible differences of these characteristics in the limbs. Thus, a systematic review will allow to verify whether or not there are asymmetries of strength and flexibility in young soccer athletes, elucidating critical points on this issue. It is worth mentioning that no revisions were found addressing this group in this specific aspect until the moment. Given the differences between young athletes and professional soccer players, it becomes relevant to consider the results separately to generate particular guidelines.

Thus, the objectives of this study were: to review the isokinetic strength assessment protocols, the values obtained for PT, and the H/Q ratio; review the unilateral evaluation protocols and normative values for flexi- bility; and verify the existence or not of asymmetries between the limbs, muscle imbalances, or BD in the strength and flexibility of young soccer players. The study hypothesized that the constant workloads generated unequally because of the dominance of limbs would influence strength and flexibility, creating asymmetries, imbalances, and BD.

\section{Material and methods}

\section{Study design}

The Preferred Reporting Items for Systematic Reviews and Meta-Analyses (PRISMA) [19] model was used for this systematic review. Studies that tried to verify asymmetry, imbalance in the $\mathrm{H} / \mathrm{Q}$ ratio, $\mathrm{BD}$ isokinetic strength and asymmetry, and BD level of unilateral flexibility in young soccer players were evaluated for possible inclusion in this systematic review.

\section{Search strategies}

Searches were carried out in the databases of PubMed, SciELO, and LILACS between September 2018 and April 2020, for articles published since 1988. The Health Sciences Descriptors from the Virtual Health Library and the Medical Subject Headings (MeSH) were used, in addition to keywords related to the research theme, divided into 3 main points:

1. Soccer, football.

2. Isokinetic, muscle strength dynamometer, muscle imbalance, muscle imbalances, strength asymmetry, strength imbalance.

3. Flexibility, joint flexibility, range of motion, range of motion articular, passive range of motion, passive straight leg raise, joint range of motion.

Within each theme, the Boolean "OR" was used, and between the intersections of topics, the Boolean "AND" was applied. Theme 1 was crossed with theme 2 , theme 1 was crossed with theme 3 , and theme 1 was crossed with themes 2 and 3 . The strategies were adopted for all databases and adjusted to the advanced search characteristics of each one. Studies with the potential to be included in the systematic review were observed by examining the citations listed in the collected publications [19]. The search strategy is detailed in Figure 1.

\section{Study inclusion and exclusion criteria}

The following inclusion criteria were used: articles with a sample of young elite male soccer players with an average age below 20 years - so that it was possible 

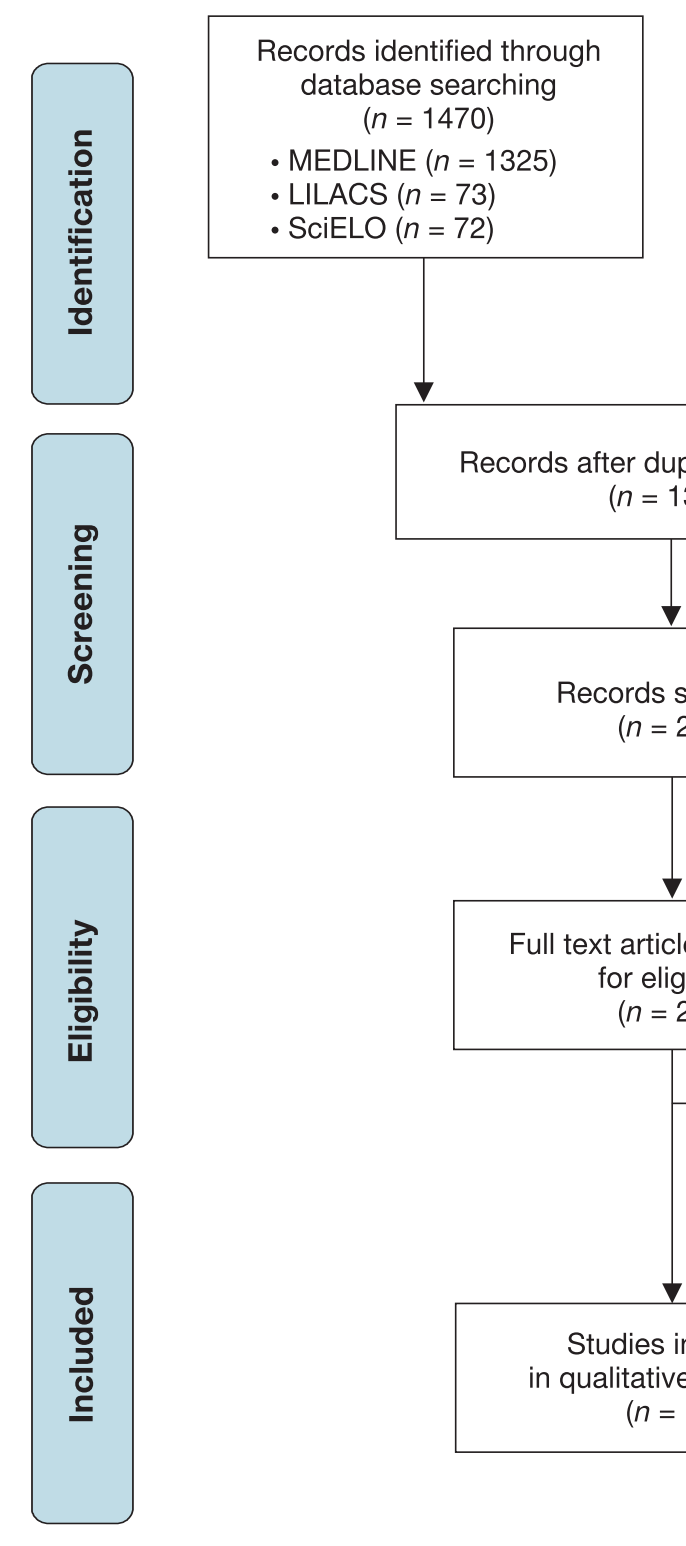

- SciELO $(n=72)$
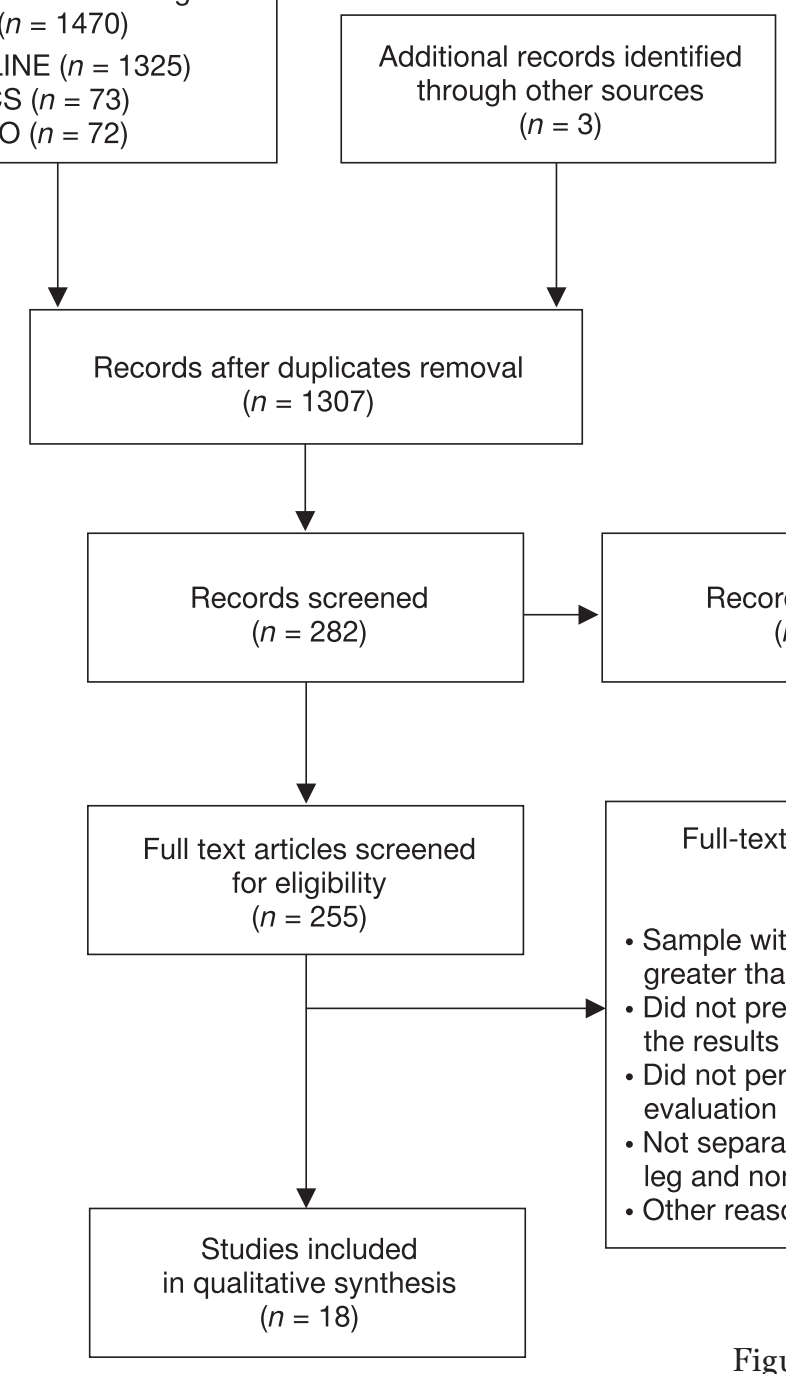

Records excluded $(n=27)$

Figure 1. Flow diagram of the different phases of the systematic review [19]

to analyse samples composed of young soccer athletes; articles in English; in the assessment of isokinetic strength: articles that evaluated the knee joint of both limbs, used speed in degrees per second and presented values for absolute PT and/or H/Q ratio; in the assessment of flexibility: articles that used tests unilaterally for the hip flexion movement and that exposed the results of both limbs - for the evaluations, these criteria were chosen so that it was possible to analyse the asymmetries and imbalances.

For exclusion, the following criteria were adopted: articles that did not separately present data related to soccer players; articles that in their sample comprised players in the process of rehabilitation; articles that did not divide limbs into DL and NDL.

\section{Study quality assessment}

Two reviewers independently assessed the quality and risk of bias concerning the results presented in each study. In any case of disagreement, a debate was held to reach a common final assessment. For this purpose, the Downs and Black [20] scale was used in an adjusted way, with the adaptation proposed by Figueiredo et al. [21]. Guiding questions were selected that included the description, internal validity, external validity (bias), and sample power of the studies. The questions were answered 'yes,' 'partially,' 'no,' or 'impossible to determine'. Only studies with a low risk of bias (score greater than or equal to 10) were included in the review. 


\section{Data extraction strategy}

In all studies, data regarding sample age and/or category of players were extracted. For studies that used isokinetic strength assessment, data were obtained concerning the test protocol, mean values and standard deviation of the absolute PT, the H/Q ratio, and, if verified, the asymmetries between the limbs and BD. For studies that assessed flexibility, the following data were extracted: instrument used, test protocol, the mean and standard deviation of the angle found for hip flexion movement, and, if verified, asymmetries between the limbs and BD. In the case of studies that performed some intervention for comparison concerning the test of isokinetic strength or flexibility, only the data from the pre-intervention moment (control moment) were extracted. From studies that in their sample presented different groups, for example, a group of young soccer players and a senior group, only data related to groups of young soccer players were extracted.

\section{Data synthesis and presentation}

The mean and standard deviation of the measures related to the assessment of isokinetic strength (PT and H/Q ratio) and flexibility (angle shown), as well as values for asymmetries and $\mathrm{BD}$ were extracted to a spreadsheet in Microsoft Excel ${ }^{\mathrm{TM}}$ (Microsoft Corporation, Santa Rosa, CA, USA). All data from the studies selected for the systematic review were extracted by the main author and reviewed by the second author.

\section{Ethical approval}

The conducted research is not related to either human or animal use.

\section{Results}

\section{Study selection}

The initial research identified 1470 studies in the selected databases, and 3 articles identified by other sources were added, totalling 1473 studies. With the removal of duplicates and articles excluded on the basis of their title and abstract, 255 articles were eligible for full verification. Subsequently, papers that did not meet the inclusion criteria were excluded. There remained 18 studies that were assessed for quality and risk of bias and added to the qualitative synthesis. Among them, 14 reported isokinetic strength, 2 demonstrated flexibility, and 2 investigated isokinetic strength and flexibility in young soccer players.

\section{Study quality assessment}

The results of the study quality assessment with the scale proposed by Downs and Black [20] adapted by Figueiredo et al. [21] are shown in Table 1 . The version

Table 1. Quality assessment results (Downs and Black scale [20] modified by Figueiredo et al. [21])

\begin{tabular}{lccccccccccccccccc}
\hline Author & 1 & 2 & 3 & 4 & 5 & 6 & 7 & 10 & 11 & 12 & 13 & 18 & 19 & 20 & 26 & 27 & Total \\
\hline Akbulut and Agopyan [3] & 1 & 1 & 1 & 1 & 1 & 1 & 1 & 1 & 0 & 0 & 1 & 1 & 1 & 1 & 0 & 0 & 12 \\
Chiamonti Bona et al. [26] & 1 & 1 & 1 & 1 & 1 & 1 & 1 & 1 & 0 & 0 & 1 & 1 & 1 & 1 & 0 & 0 & 12 \\
Costa Silva et al. [7] & 1 & 1 & 1 & 1 & 1 & 1 & 1 & 1 & 0 & 0 & 1 & 1 & 1 & 1 & 0 & 1 & 13 \\
Daneshjoo et al. [24] & 1 & 1 & 1 & 1 & 2 & 1 & 1 & 1 & 0 & 0 & 1 & 1 & 1 & 1 & 0 & 0 & 13 \\
Daneshjoo et al. [13] & 1 & 1 & 1 & 1 & 2 & 1 & 1 & 1 & 0 & 0 & 1 & 1 & 1 & 1 & 0 & 0 & 13 \\
Daneshjoo et al. [2] & 1 & 1 & 1 & 1 & 1 & 1 & 1 & 1 & 0 & 0 & 1 & 1 & 1 & 1 & 0 & 0 & 12 \\
Eustace et al. [27] & 1 & 1 & 1 & 1 & 1 & 1 & 1 & 1 & 0 & 0 & 1 & 1 & 1 & 1 & 0 & 1 & 13 \\
Forbes et al. [22] & 1 & 1 & 1 & 1 & 1 & 1 & 1 & 1 & 0 & 0 & 1 & 1 & 1 & 1 & 1 & 0 & 13 \\
Gür et al. [11] & 1 & 1 & 1 & 1 & 1 & 1 & 1 & 0 & 0 & 0 & 1 & 1 & 1 & 1 & 0 & 0 & 11 \\
Harper et al. [28] & 1 & 1 & 1 & 1 & 1 & 1 & 1 & 0 & 0 & 0 & 1 & 1 & 1 & 1 & 0 & 0 & 11 \\
Lehance et al. [23] & 1 & 1 & 1 & 1 & 1 & 1 & 1 & 0 & 0 & 0 & 1 & 1 & 1 & 1 & 0 & 0 & 11 \\
Lehnert et al. [25] & 1 & 1 & 1 & 1 & 1 & 1 & 1 & 0 & 0 & 0 & 1 & 1 & 1 & 1 & 0 & 0 & 11 \\
De Lira et al. [8] & 1 & 1 & 1 & 1 & 1 & 1 & 1 & 1 & 0 & 0 & 1 & 1 & 1 & 1 & 0 & 0 & 12 \\
Maly et al. [10] & 1 & 1 & 1 & 1 & 1 & 1 & 1 & 0 & 0 & 0 & 1 & 1 & 1 & 1 & 0 & 0 & 11 \\
Ramos et al. [16] & 1 & 1 & 1 & 1 & 1 & 1 & 1 & 1 & 0 & 0 & 1 & 1 & 1 & 1 & 0 & 0 & 12 \\
Sanz et al. [15] & 1 & 1 & 1 & 1 & 1 & 1 & 1 & 1 & 0 & 0 & 1 & 1 & 1 & 1 & 0 & 0 & 12 \\
Seabra et al. [12] & 1 & 1 & 1 & 1 & 2 & 1 & 1 & 1 & 0 & 0 & 1 & 1 & 1 & 1 & 0 & 0 & 13 \\
Slimowski et al. [9] & 1 & 1 & 1 & 1 & 2 & 1 & 1 & 1 & 0 & 0 & 1 & 1 & 1 & 1 & 0 & 0 & 13 \\
\hline
\end{tabular}

For items 1-4, 6-7, and 10: 0 = no, 1 = yes; item 5: 0 = no, 1 = partially, 2 = yes; items $11-13,18-20$, 26: 0 = no/impossible to determine, 1 = yes; item 27 : the score could range $0-5$ 
used employed 16 of the 27 items from the original text. Therefore, the lowest score assumed to consider the study as being of high quality was 10 [21]. Most studies (13 articles) were within the score range of 12-13, and all studies obtained a high-quality score $(\geq 10)$.

\section{Strength}

Table 2 illustrates the main characteristics of the studies included $(n=16)$ in the qualitative analysis concerning the use and protocol of the isokinetic strength test in young soccer players. The protocols applied in the test were highlighted, with the consideration of the verified muscular action, the evaluated speeds, and repetitions. All these characteristics suffered divergences among the studies found. Among the analysed variables, the most used were the concentric muscular actions of the quadriceps and hamstrings ( $n=16, n=15$, respectively) [2, 7-13, 16, 22-28], the speeds of $60 \%$ s $(n=13)[2,8-10,13,16,22-28]$ and $180^{\circ} \mathrm{s}(n=9)[2,7,10,11,13,16,24,27,28]$; in relation to the repetition maximum (RM) performed, we found a variation of 3-5 among the studies.

Table 3 presents all the studies included in the qualitative synthesis that reported isokinetic strength $(n=16)$ and their results concerning asymmetries and/or strength BD. Among the inserted studies that verified asymmetry concerning PT $(n=8), 6$ articles did not reveal significant asymmetries between DL and NDL $[2,7,10-13]$. Of the articles that found asymmetry concerning the $\mathrm{H} / \mathrm{Q}$ ratio between DL and NDL $(n=7)$, 6 studies did not observe a significant result $[2,7,8$, $10,11,24]$. Only 9 studies analysed possible BD, and the results were divergent: 5 articles did not find significant BD [7-9, 23, 26], while the other 4 articles reported the presence of significant BD [2, 10, 16, 22]. In the $\mathrm{BD}$, it was possible to verify other points of mismatch, such as the value considered as the cut-off point, which varied between $>10 \%$ and $>15 \%$ (this, however, did not influence the studies that found or did not find a significant result), and the way it was calculated. Not all studies verified these characteristics, and some did not make it clear whether they were evaluated and/or found.

Table 4 presents the values (mean \pm standard deviation) of the absolute PT $(\mathrm{N} \cdot \mathrm{m})$ depending on the age group, muscular action, limb, and evaluated speed. These data were extracted from 13 studies inserted in the qualitative synthesis $[2,7-9,11-13,16,22$, 25-28]. A total of 590 individuals were evaluated, whose age ranged 11-20 years. The assessed actions were the concentric and/or eccentric quadriceps and/or hamstrings at different speeds $(30 \%, 60 \%, 90 \%$, $120 \%$ s, $180 \%$ s, $240 \%$ s, $270 \%$ s, $300 \%$ s). Combining the age variation with the differences in the actions and speeds used in the tests, one can observe an oscillation of the PT found in the studies.

Table 2. Studies inserted in the qualitative analysis with the protocols used in the isokinetic test for muscular action, with evaluated speeds and repetitions $(n=16)$

\begin{tabular}{|c|c|c|c|}
\hline Author & Actions & Angular velocities & Test \\
\hline Gür et al. [11] & $\mathrm{Q}$ and $\mathrm{H}$ Conc and Ecc & $30 \% \mathrm{~s}-180 \% \mathrm{~s}-240 \% \mathrm{~s}-300 \% \mathrm{~s}$ & $4 \mathrm{RM}$ \\
\hline Forbes et al. [22] & $\mathrm{Q}$ and $\mathrm{H}$ Conc $+\mathrm{H}$ Ecc & $60 \% \mathrm{~s}$ & $5 \mathrm{RM}$ \\
\hline Lehance et al. [23] & $\mathrm{Q}$ and H Conc (1) + H Ecc (2) & $60 \%$ s $-240 \%$ s (1); 30\% $-120 \%$ s (2) & - \\
\hline Seabra et al. [12] & $\mathrm{Q}$ and $\mathrm{H}$ Conc & $90 \% \mathrm{~s}$ & $3 \mathrm{RM}$ \\
\hline Daneshjoo et al. [24] & $\mathrm{Q}$ and H Conc (1) + H Ecc (2) & $60 \%$ s $-180 \%$ s $-300 \%$ s (1); $120 \%$ s (2) & $3 \mathrm{RM}$ \\
\hline \multicolumn{4}{|l|}{ Daneshjoo et al. [13] } \\
\hline Daneshjoo et al. [2] & $\mathrm{Q}$ and $\mathrm{H}$ Conc & $60 \% \mathrm{~s}-180 \% \mathrm{~s}-300 \% \mathrm{~s}$ & $3 \mathrm{RM}$ \\
\hline Lehnert et al. [25] & $\mathrm{Q}$ and $\mathrm{H}$ Conc and Ecc & $60 \%$ & $6 \mathrm{RM}$ \\
\hline Costa Silva et al. [7] & $\mathrm{Q}$ and $\mathrm{H}$ Conc and Ecc & $180 \% \mathrm{~s}$ & $5 \mathrm{RM}$ \\
\hline Śliwowski et al. [9] & $\mathrm{Q}$ and $\mathrm{H}$ Conc & $60 \%$ & $3 \mathrm{RM}$ \\
\hline Maly et al. [10] & $\mathrm{Q}$ and $\mathrm{H}$ Conc & $60 \% \mathrm{~s}-120 \% \mathrm{~s}-180 \% \mathrm{~s}-240 \% \mathrm{~s}-300 \% \mathrm{~s}$ & $3 \mathrm{RM}$ \\
\hline Chiamonti Bona et al. [26] & $\mathrm{Q}$ and $\mathrm{H}$ Conc & $60 \% \mathrm{~s}(1)-300 \% \mathrm{~s}(2)$ & $\begin{array}{r}5 \text { RM (1) } \\
20 \text { RM (2) }\end{array}$ \\
\hline De Lira et al. [8] & $\mathrm{Q}$ and $\mathrm{H}$ Conc & $60 \%$ & $5 \mathrm{RM}$ \\
\hline Eustace et al. [27] & $\mathrm{Q}$ Conc $+\mathrm{H}$ Ecc & $60 \% \mathrm{~s}-180 \% \mathrm{~s}-270 \% \mathrm{~s}$ & $3 \mathrm{RM}$ \\
\hline Harper et al. [28] & $\mathrm{Q}$ and $\mathrm{H}$ Conc and Ecc & $60 \%-180 \% \mathrm{~s}$ & $5 \mathrm{RM}$ \\
\hline Ramos et al. [16] & $\mathrm{Q}$ and $\mathrm{H}$ Conc $+\mathrm{H} \mathrm{Ecc}$ & $60 \% \mathrm{~s}-180 \% \mathrm{~s}$ & $5 \mathrm{RM}$ \\
\hline
\end{tabular}

Conc - concentric, Ecc - eccentric, H - hamstrings, Q - quadriceps, RM - repetition maximum 
S. Ramos et al., Asymmetries in young soccer players: a review

Table 3. Studies inserted in the qualitative synthesis on the isokinetic test in accordance with asymmetries and BD $(n=16)$

\begin{tabular}{|c|c|c|c|c|}
\hline Author & PT asymmetries & $\begin{array}{c}\mathrm{H} / \mathrm{Q} \text { ratio } \\
\text { asymmetries* }\end{array}$ & $\mathrm{BD}$ & Observations on BD \\
\hline Gür et al. [11] & No & No & Not verified & - \\
\hline $\begin{array}{l}\text { Forbes } \\
\text { et al. [22] }\end{array}$ & It is not clear & It is not clear & $\begin{array}{l}\text { Yes. Sub- } 12 \text { showed greater } \\
\text { inequality between members } \\
(1.2 \text { - suggesting a stronger DL } \\
\text { for the concentric action of } \mathrm{H})\end{array}$ & $\begin{array}{l}\text { Bilateral asymmetry = } \\
\text { PT DL/PT NDL }\end{array}$ \\
\hline $\begin{array}{l}\text { Lehance } \\
\text { et al. [23] }\end{array}$ & It is not clear & It is not clear & $\begin{array}{l}\text { It was not significant. However, } \\
\text { the players who presented showed } \\
\text { a predominance in the eccentric } \\
\text { bilateral asymmetry of } \mathrm{H}\end{array}$ & $\begin{array}{l}\text { Bilateral comparison: } \\
\text { asymmetries in percentages. } \\
\text { Cut-off point: over } 15 \%\end{array}$ \\
\hline Seabra et al. [12] & No & - & Not verified & - \\
\hline $\begin{array}{l}\text { Daneshjoo et al. [24] } \\
\text { Daneshjoo et al. [13] }\end{array}$ & No & No & Not verified & - \\
\hline Daneshjoo et al. [2] & No & No & $\begin{array}{l}\text { Yes. In } 97.2 \% \text { of the sample, mainly } \\
\text { in the concentric action of } \mathrm{H}\end{array}$ & $\begin{array}{l}\text { Strength deficit: difference } \\
\text { between the strength of the } \\
\text { muscles in the opposite extrem- } \\
\text { ities. Cut-off point: over } 10 \%\end{array}$ \\
\hline
\end{tabular}

\begin{tabular}{lccc}
\hline $\begin{array}{l}\text { Lehnert } \\
\text { et al. [25] }\end{array}$ & It is not clear & It is not clear & Not verified \\
\hline $\begin{array}{l}\text { Costa Silva } \\
\text { et al. [7] }\end{array}$ & No & No & Not significant
\end{tabular}

ities. Cut-off point: over $10 \%$

Śliwowski et al. [9] It is not clear It is not clear Not significant

\begin{tabular}{llll}
\hline Maly et al. [10] & No & Yes. $60 \%$ s: $51 \%$ of the players & Bilateral asymmetry $=$ \\
& & and $300 \%$ s: $41 \%$ of the players & PT Q/PT Q; PT H/PT H. \\
& presented an asymmetry for H; & Cut-off point: over $10 \%$
\end{tabular}
asymmetry between the limbs. Tendency for NDL to be more deficient, but it was not significant

\begin{tabular}{|c|c|c|c|c|}
\hline $\begin{array}{l}\text { Chiamonti Bona } \\
\text { et al. [26] }\end{array}$ & $\begin{array}{c}\text { Yes. Sub- } 15 \text { at } \\
60 \% \text { for concentric } \\
\text { action of } \mathrm{Q}, \\
\text { stronger NDL }\end{array}$ & & Not significant & Cut-off point: over $10-15 \%$ \\
\hline
\end{tabular}

\begin{tabular}{|c|c|c|c|c|}
\hline De Lira et al. [8] & No & No & $\begin{array}{l}\text { Not significant. } 14 \% \text { of the } \\
\text { athletes presented for } Q \\
\text { and } 18 \% \text { of the players for } H\end{array}$ & $\begin{array}{l}\text { Bilateral strength deficit }= \\
((\mathrm{PT} \text { DL - PT NDL) / PT DL) } \times \\
\text { 100. Cut-off point: over } 15 \%\end{array}$ \\
\hline Eustace et al. [27] & It is not clear & It is not clear & Not verified & $\begin{array}{l}\text { Another type of asymmetry } \\
\text { found, which used the angle } \\
\text { of movement }\end{array}$ \\
\hline
\end{tabular}

\begin{tabular}{lccll}
\hline Harper et al. [28] & It is not clear & - & Not verified & - \\
\hline Ramos et al. [16] & Yes. DL signifi- & Yes. DL signifi- & Yes. 57\% of the players presented & Cut-off point: over 15\% \\
& cantly stronger at & cantly more & to H in the eccentric action & \\
& $\begin{array}{c}60 \% \text { s in the eccen- } \\
\text { tric action of H }\end{array}$ & balanced & & \\
& & & \\
\hline
\end{tabular}

BD - bilateral differences, DL - dominant limb, H - hamstrings, NDL - non-dominant limb, PT - peak torque, Q - quadriceps * asymmetry between DL and NDL for the H/Q ratio 


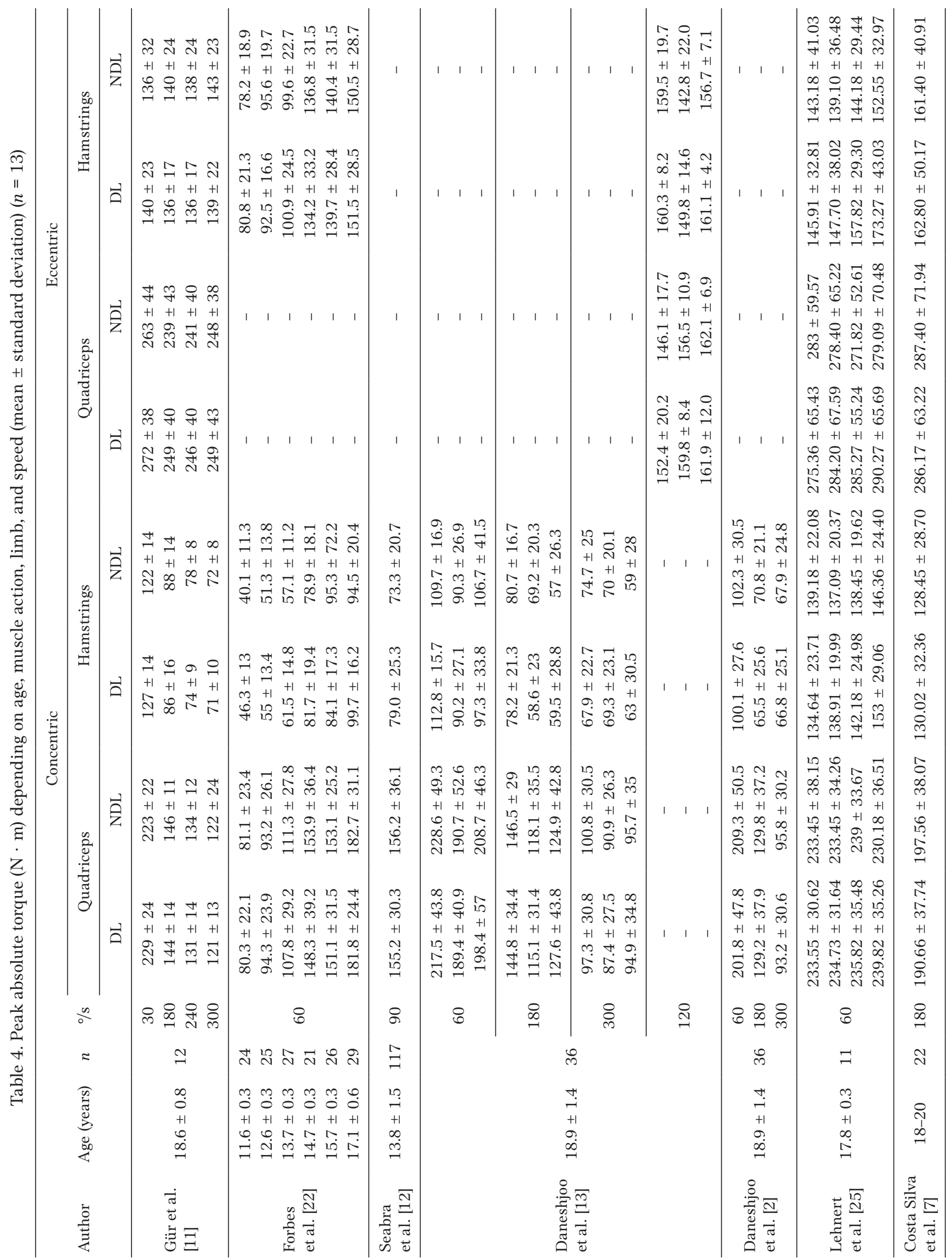


Table 5 shows the values concerning the H/Q ratio depending on the age range, limb, and type of ratio evaluated found in 12 studies [2, 7-11, 16, 22-25, 27]. Forbes et al. [22] and Lehnert et al. [25] presented the data in the form of variation and approximate values; in all other studies, it was possible to extract the mean \pm standard deviation for the H/Q ratio. A total of 489 individuals were evaluated, whose age ranged 11-20 years. The ratios found were (in the order of most to least used): conventional H/Q at $30 \%, 60 \%$, $120 \%$ s, $180 \%$ s, $240 \%$ s, and $300 \%$ s [2, 7-11, 16, 22-25]; functional H/Q at $30 \%$ s, $60 \%$ s, $120 \%$ s, $180 \%$ s, $240 \%$, $270 \%$, and $300 \%$ s [7, 11, 16, 22, 24, 25, 27]; functional hamstrings $30 \%$ s quadriceps $240 \%$ [23]; eccentric H/Q at $30 \%$ s, $180 \%$ s, $240 \%$ s, and $300 \%$ [ [11]; fast/slow, which consisted of the quadriceps concentric action at $300 \%$ by the same action at $60 \%$, and the same was performed for the hamstrings [24].

\section{Flexibility}

Table 6 illustrates the main characteristics of the studies included $(n=4)$ in the qualitative synthesis concerning the use, test protocol, and results of flexibility unilateral assessment in young soccer players. A total of 1747 individuals aged 8-20 years were evaluated. The goniometry technique was mostly applied for evaluation [2, 3, 16]; however, the protocol to assess the method was divergent among all studies. Of the 3 articles that found asymmetry, 2 reported DL significantly more flexible than NDL $[2,15]$. Only Ramos et al. [16] observed BD and did not establish an expressive result.

\section{Strength and flexibility}

Only Daneshjoo et al. [2] and Ramos et al. [16] observed possible interactions between strength and flexibility. The results found by these authors can be checked in Tables 2-6 separately for strength and flexibility. Neither of these studies managed to establish a relationship between these characteristics, with divergent results.

\section{Discussion}

The present systematic literature review sought to characterize asymmetries of isokinetic strength and flexibility in young soccer players. The focus of our analysis was the use of the isokinetic test to assess muscle strength and to assess flexibility unilaterally, for a possible comparison between DL and NDL. The 
Table 5. H/Q ratio presented in each study, depending on the age group, limb, and type of ratio evaluated $(n=12)$

\begin{tabular}{|c|c|c|c|c|c|c|c|c|c|c|c|}
\hline \multirow{2}{*}{ Author } & \multirow{2}{*}{$\begin{array}{c}\text { Age } \\
\text { (years) }\end{array}$} & \multirow{2}{*}{$n$} & \multirow{2}{*}{$\%$ s } & \multicolumn{2}{|c|}{ Conventional ratio } & \multicolumn{2}{|c|}{ Functional ratio } & \multicolumn{2}{|c|}{ Eccentric ratio } & \multicolumn{2}{|c|}{ Fast/slow ratio } \\
\hline & & & & DL & NDL & DL & NDL & DL & NDL & DL & NDL \\
\hline \multirow{4}{*}{$\begin{array}{l}\text { Gür } \\
\text { et al. [11] }\end{array}$} & \multirow{4}{*}{$18.6 \pm 0.8$} & \multirow{4}{*}{12} & 30 & $0.57 \pm 0.06$ & $0.56 \pm 0.08$ & $0.62 \pm 0.11$ & $0.59 \pm 0.14$ & $0.52 \pm 0.08$ & $0.53 \pm 0.10$ & - & - \\
\hline & & & 180 & $0.60 \pm 0.09$ & $0.61 \pm 0.09$ & $0.94 \pm 0.18$ & $0.97 \pm 0.22$ & $0.55 \pm 0.09$ & $0.58 \pm 0.12$ & - & - \\
\hline & & & 240 & $0.58 \pm 0.06$ & $0.60 \pm 0.06$ & $1.13 \pm 0.35$ & $1.06 \pm 0.20$ & $0.58 \pm 0.10$ & $0.60 \pm 0.15$ & - & - \\
\hline & & & 300 & $0.60 \pm 0.06$ & $0.62 \pm 0.08$ & $1.19 \pm 0.26$ & $1.14 \pm 0.19$ & $0.57 \pm 0.09$ & $0.59 \pm 0.14$ & - & - \\
\hline \multirow{6}{*}{$\begin{array}{l}\text { Forbes } \\
\text { et al. [22] }\end{array}$} & $11.6 \pm 0.3$ & 24 & \multirow{6}{*}{60} & \multirow{6}{*}{\multicolumn{2}{|c|}{$0.50-0.62$}} & \multirow{6}{*}{\multicolumn{2}{|c|}{$1.10-0.85$}} & - & - & - & - \\
\hline & $12.6 \pm 0.3$ & 25 & & & & & & - & - & - & - \\
\hline & $13.7 \pm 0.3$ & 27 & & & & & & _- & _- & _- & _- \\
\hline & $14.7 \pm 0.3$ & 21 & & & & & & - & - & - & - \\
\hline & $15.7 \pm 0.3$ & 26 & & & & & & - & - & - & - \\
\hline & $17.1 \pm 0.6$ & 29 & & & & & & - & - & - & - \\
\hline \multirow{6}{*}{$\begin{array}{l}\text { Lehance } \\
\text { et al. [23] }\end{array}$} & & & 60 & $0.63 \pm 0.07$ & $0.61 \pm 0.08$ & - & - & - & - & - & - \\
\hline & $15.7 \pm 0.8$ & 18 & 240 & $0.74 \pm 0.15$ & $0.73 \pm 0.13$ & - & - & - & - & - & - \\
\hline & & & $30: 240$ & - & - & - & $1.49 \pm 0.31$ & - & - & - & - \\
\hline & & & 60 & $0.60 \pm 0.07$ & $0.61 \pm 0.08$ & - & - & - & - & - & - \\
\hline & $19.5 \pm 1.6$ & 20 & 240 & $0.73 \pm 0.12$ & $0.75 \pm 0.17$ & - & - & - & - & - & - \\
\hline & & & $30: 240$ & - & - & $1.50 \pm 0.29$ & $1.48 \pm 0.26$ & - & - & - & - \\
\hline & & & & $0.53 \pm 0.8$ & $0.50 \pm 0.1$ & - & - & - & - & - & - \\
\hline & & & 60 & $0.48 \pm 0.1$ & $0.49 \pm 0.2$ & - & - & - & - & - & - \\
\hline & & & & $0.49 \pm 0.1$ & $0.51 \pm 0.1$ & - & - & - & - & - & - \\
\hline & & & & - & - & $0.86 \pm 0.5$ & $0.82 \pm 0.4$ & - & - & - & - \\
\hline & & & 120 & - & - & $0.66 \pm 0.2$ & $0.72 \pm 0.4$ & - & - & - & - \\
\hline & & & & - & - & $0.69 \pm 0.2$ & $0.70 \pm 0.2$ & - & - & - & - \\
\hline & & & & $0.54 \pm 0.9$ & $0.56 \pm 0.1$ & - & - & - & - & - & - \\
\hline & & & 180 & $0.51 \pm 0.1$ & $0.61 \pm 0.2$ & - & - & - & - & - & - \\
\hline Daneshjoo & $18.9 \pm 1.4$ & 36 & & $0.47 \pm 0.2$ & $0.51 \pm 0.1$ & - & - & - & - & - & - \\
\hline & & & & $0.72 \pm 0.2$ & $0.75 \pm 0.2$ & - & - & - & - & - & - \\
\hline & & & 300 & $0.83 \pm 0.2$ & $0.81 \pm 0.2$ & - & - & - & - & - & - \\
\hline & & & & $0.68 \pm 0.3$ & $0.67 \pm 0.4$ & - & - & - & - & - & - \\
\hline & & & & - & - & - & - & - & - & $0.45 \pm 0.1$ & $0.45 \pm 2$ \\
\hline & & & 300: & - & - & - & - & - & - & $0.46 \pm 0.1$ & $0.48 \pm 0.1$ \\
\hline & & & & - & - & - & - & - & - & $0.48 \pm 0.1$ & $0.46 \pm 0.1$ \\
\hline & & & 300 & - & - & - & - & - & - & $0.60 \pm 0.2$ & $0.68 \pm 0.2$ \\
\hline & & & 300: & - & - & - & _- & _- & - & $0.79 \pm 0.2$ & $0.79 \pm 0.2$ \\
\hline & & & & - & - & - & - & - & - & $0.69 \pm 0.3$ & $0.58 \pm 0.2$ \\
\hline & & & 60 & $0.50 \pm 0.11$ & $0.50 \pm 0.14$ & - & - & - & - & - & - \\
\hline Daneshjoo & $18.9 \pm 1.4$ & 36 & 180 & $0.51 \pm 0.13$ & $0.56 \pm 0.14$ & - & - & - & - & - & - \\
\hline & & & 300 & $0.74 \pm 0.22$ & $0.75 \pm 0.26$ & - & - & - & - & - & - \\
\hline $\begin{array}{l}\text { Lehnert } \\
\text { et al. [25] }\end{array}$ & $17.8 \pm 0.3$ & 11 & 60 & ca. 0 & 0.60 & 0.60 & -0.70 & - & - & - & - \\
\hline $\begin{array}{l}\text { Costa Silva } \\
\text { et al. [7] }\end{array}$ & $18-20$ & 22 & 180 & $0.68 \pm 0.14$ & $0.65 \pm 0.10$ & $0.86 \pm 0.26$ & $0.82 \pm 0.17$ & - & - & - & - \\
\hline Śliwowski & & & & $0.60 \pm 0.03$ & $0.58 \pm 0.03$ & - & - & - & - & - & - \\
\hline et al. [9] & $17-18$ & 24 & 60 & $0.53 \pm 0.07$ & $0.51 \pm 0.06$ & - & - & - & - & - & - \\
\hline & & & 60 & $0.59 \pm 0.08$ & $0.56 \pm 0.08$ & - & - & - & - & - & - \\
\hline & & & 120 & $0.62 \pm 0.08$ & $0.59 \pm 0.09$ & - & - & - & - & - & - \\
\hline Maly & $15.7 \pm 0.3$ & 41 & 180 & $0.63 \pm 0.09$ & $0.60 \pm 0.10$ & - & - & - & - & - & - \\
\hline & & & 240 & $0.63 \pm 0.10$ & $0.60 \pm 0.11$ & - & - & - & - & - & - \\
\hline & & & 300 & $0.63 \pm 0.12$ & $0.60 \pm 0.13$ & - & - & - & - & - & - \\
\hline $\begin{array}{l}\text { De Lira } \\
\text { et al. [8] }\end{array}$ & $19.3 \pm 4.8$ & 70 & 60 & $0.53 \pm 0.09$ & $0.53 \pm 0.07$ & - & - & - & - & - & - \\
\hline & & & 60 & - & - & $0.73 \pm 0.17$ & $0.72 \pm 0.17$ & - & - & - & - \\
\hline Eustace & $17.0 \pm 0.6$ & 17 & 180 & - & - & $0.98 \pm 0.19$ & $0.95 \pm 0.16$ & - & - & - & - \\
\hline et al. [27] & & & 270 & - & - & $1.19 \pm 0.32$ & $1.16 \pm 0.21$ & - & - & - & - \\
\hline Ramos & & & 60 & $52.94 \pm 11.38$ & $55.19 \pm 9.38$ & $69.26 \pm 12.34$ & $61.13 \pm 11.41$ & - & - & - & - \\
\hline et al. [16] & 15 & 30 & 180 & $65 \pm 10.98$ & $63.58 \pm 12.62$ & $79 \pm 18.49$ & $72.94 \pm 17.11$ & - & - & - & - \\
\hline
\end{tabular}

DL - dominant limb, H - hamstrings, NDL - non-dominant limb, Q - quadriceps 
Table 6. Studies inserted in the qualitative synthesis concerning flexibility, with evaluation protocol, result, asymmetry, and $\operatorname{BD}(n=4)$

\begin{tabular}{|c|c|c|c|c|c|c|c|}
\hline \multirow{2}{*}{ Author } & \multirow{2}{*}{ Sample } & \multirow{2}{*}{ Evaluation protocol } & \multicolumn{3}{|c|}{$\operatorname{Result}\left({ }^{\circ}\right)$} & \multirow{2}{*}{ Asymmetry } & \multirow{2}{*}{$\mathrm{BD}$} \\
\hline & & & & DL & NDL & & \\
\hline $\begin{array}{l}\text { Daneshjoo } \\
\text { et al. [2] }\end{array}$ & $\begin{array}{c}n=36 \\
(18.9 \pm 1.4)\end{array}$ & $\begin{array}{l}\text { Without warming up; } 3 \text { measure- } \\
\text { ments for each limb for hip flexion } \\
\text { through goniometry; mean values } \\
\text { were used }\end{array}$ & & $108.8 \pm 10.7$ & $104.6 \pm 9.8$ & $\begin{array}{l}\text { Yes. DL signifi- } \\
\text { cantly more } \\
\text { flexible } \\
\text { than NDL }\end{array}$ & Not verified \\
\hline $\begin{array}{l}\text { Akbulut } \\
\text { and } \\
\text { Agopyan } \\
\text { [3] }\end{array}$ & $\begin{array}{c}n=24 \\
(15.6 \pm 0.4)\end{array}$ & $\begin{array}{l}\text { Without warming up; } 2 \text { measure- } \\
\text { ments for each limb for hip flexion } \\
\text { through goniometry; higher value } \\
\text { was used }\end{array}$ & $\begin{array}{l}\text { Gr } 1 \\
\text { Gr } 2\end{array}$ & $\begin{array}{l}58.5 \pm 9.1 \\
60.0 \pm 9.6\end{array}$ & $\begin{array}{l}59.7 \pm 10.1 \\
59.1 \pm 8.4\end{array}$ & Not verified & Not verified \\
\hline $\begin{array}{l}\text { Ramos } \\
\text { et al. [16] }\end{array}$ & $\begin{array}{c}n=30 \\
(15.97 \pm 0.67)\end{array}$ & $\begin{array}{l}\text { Warming up: } 2 \text { series of static } \\
\text { stretching, maintaining the position } \\
\text { for } 10 \text { seconds, in } 4 \text { different move- } \\
\text { ments for the lower limbs; } 3 \text { attempts } \\
\text { for each limb for hip flexion through } \\
\text { goniometry; higher value was used }\end{array}$ & & $100.1 \pm 8.82$ & $100.4 \pm 10.44$ & No & $\begin{array}{l}\text { No. Only } 17 \% \\
\text { of athletes with } \\
\text { BD }>10 \%\end{array}$ \\
\hline $\begin{array}{l}\text { Sanz et al. } \\
{[15]}\end{array}$ & $\begin{array}{c}n=1657 \\
(12.58 \pm 2.65)\end{array}$ & $\begin{array}{l}\text { Without warming up; } 2 \text { measure- } \\
\text { ments for each limb for hip flexion } \\
\text { through the active leg elevation } \\
\text { test that was filmed and analysed } \\
\text { with the Kinovea software; higher } \\
\text { value was used }\end{array}$ & $\begin{array}{l}\text { U9 } \\
\text { U11 } \\
\text { U13 } \\
\text { U15 } \\
\text { U18 }\end{array}$ & $\begin{array}{l}57.85 \pm 8.03 \\
56.01 \pm 8.40 \\
54.05 \pm 8.14 \\
56.60 \pm 8.75 \\
57.82 \pm 9.67\end{array}$ & $\begin{array}{l}56.43 \pm 7.88 \\
54.49 \pm 8.44 \\
52.90 \pm 8.33 \\
55.25 \pm 8.64 \\
56.69 \pm 9.78\end{array}$ & $\begin{array}{l}\text { Yes. DL signifi- } \\
\text { cantly more } \\
\text { flexible } \\
\text { than NDL } \\
\text { for all ages }\end{array}$ & Not verified \\
\hline
\end{tabular}

BD - bilateral differences, DL - dominant limb, Gr - group, NDL - non-dominant limb

initial hypothesis was partially rejected, as our main findings indicate a symmetrical pattern for PT and the $\mathrm{H} / \mathrm{Q}$ ratio between limbs, divergent results for $\mathrm{BD}$ for strength, and a possible trend, which needs to be further investigated, to flexibility asymmetry, with a more flexible DL than NDL.

Regarding the asymmetries of $\mathrm{PT}$ and the H/Q ratio among the athletes, of the articles that analysed them, the majority did not find any statistically significant differences [2, 7, 8, 10-13, 24]. However, in the papers that evaluated $\mathrm{BD}$, the observed results were divergent. In this case, the very definition of $\mathrm{BD}$ and the way it was verified were also heterogeneous, which can influence the results. In this specific situation, we suggest the use of the cut-off point for values higher than $15 \%$, on the basis of prospective studies in professional soccer players which revealed a higher injury risk in individuals who presented these BD [5, 29].

From the verified results, it seems reasonable to suggest that even with a preference among the participants and the existence of dominance for strength, soccer training does not generate an asymmetric DL and NDL development in young players, a characteristic that can be corroborated in players who are at the beginning of their sports career [12] and have not yet been exposed to so many training/game asymmetric loads.
Another factor is the growth of new training cultures, such as the incentive to use both legs and the implementation of and greater adherence to strength training within the modality, especially in these initial years $[7,25]$. Besides, our results are in line with those obtained by DeLang et al. [30] through a meta-analysis; they also did not find strength asymmetries or imbalances for the H/Q ratio between limbs in football players of different ages and levels.

It was possible to verify the assessment of isokinetic strength in young soccer players. A wide variety of protocols for the test makes it difficult to compare the data extracted from the studies. It becomes necessary to introduce standardization, or for the tests to be more homogeneous. In the analysed studies, the following were suggested as a test protocol: concerning the action - check the concentric action of quadriceps and hamstrings and the eccentric action of hamstrings; concerning speed $-60 \%$ and $180 \%$ s; concerning RM between 3 and 5 RM. Through the obtained data, we recommend the verification of the conventional and functional ratio, asymmetries concerning PT and the $\mathrm{H} / \mathrm{Q}$ ratio between DL and NDL, imbalances of the $\mathrm{H} / \mathrm{Q}$ ratio, and $\mathrm{BD}$.

Taking into account the data previously suggested for the evaluation protocol, one can observe an increase 
in PT values with increasing age; also, higher values were found at $60 \%$ when compared with $180 \%$ s [2, 7-9, 11, 13, 16, 22, 25-28]. Regarding the values for the $\mathrm{H} / \mathrm{Q}$ ratio, age and speed did not seem to influence the results. The values orbited around 0.60 for the conventional ratio at $60 \%$ and $180 \%$ s $[2,7-11,16$, 22-25] and near 0.90 for the functional ratio at $60 \%$ and $180 \%$ s [7, 11, 16, 22, 25, 27]. The same results are assumed for adult soccer players, so age will not influence the expected response to the H/Q ratio [31].

It is important to monitor these characteristics because for asymmetries exceeding the norms, interventions are necessary to restore a symmetrical pattern and, consequently, reduce the risk of injuries. It is worth highlighting the importance of strength training and its use within the sport. It is an effective intervention to improve performance (increase the jump, ability to change direction, strength; improve running; etc.) and the best method to prevent injuries. It constitutes part of the rehabilitation process [32, 33] and serves to reduce, in a targeted way, possible asymmetries, imbalances, or BD of strength [9].

Regarding flexibility, there is a scarcity of studies that verified it unilaterally. Among the papers inserted that examined possible asymmetries, Daneshjoo et al. [2] and Sanz et al. [15] found DL significantly more flexible than NDL, which can be understood as a tendency towards an asymmetry between the limbs. This can be corroborated by the fact that flexibility may not develop in the same way as strength, even though the other limb is used as a support when kicking. Besides, stretching exercises, traditionally used within the sport, may not be enough to improve this valence. In this case, specific protocols, such as the proprioceptive neuromuscular facilitation method, would be needed to increase the range of motion of the lower limbs and, consequently, kick speed [3].

To perform this monitoring, flexibility assessment through goniometry is suggested. Goniometry is a simple, fast, inexpensive, reliable, and valid technique for measuring the flexibility of the posterior chain of the lower limbs [34], for the movement of hip flexion. Regarding normative values, Witvrouw et al. [35], evaluating adult players, found that athletes who presented a smaller angle of $90^{\circ}$, as a result, had a significantly higher risk of injuries. Owing to the lack of studies among young athletes, we suggest adopting this value as a reference. It is also necessary to monitor possible BD. Because of the scarcity of studies included in the qualitative synthesis, it is not possible to state that the asymmetry of flexibility is natural to the sports modality.
As for the interaction between strength and flexibility, Ramos et al. [16] and Daneshjoo et al. [2] found no results that could establish a relationship between these characteristics. In the former article, a significant difference was observed only for strength; in the latter study, DL was significantly more flexible than NDL and no significant difference in strength was reported. These conflicting results can be explained by differences in the methodologies to verify these valences. However, Fousekis et al. [5], who assessed strength and flexibility in adult soccer players, suggested that an association of asymmetries in these attributes might enhance mechanisms generating muscle injuries. Ribeiro-Alvares et al. [36] highlight that low eccentric strength in the hamstrings will be one of the main risk factors for injury and that it may be associated with, for example, flexibility deficits, increasing the injury risk.

The main limitation of the analysis is the significant divergence in the assessment protocols for strength and flexibility. It is also necessary to reflect on the forms of warming up that can be used, since these can influence the results [37]; therefore, a detailed description of how this part was performed becomes significant. More specifically, for flexibility, there is still a difficulty in applying tests unilaterally. We would thus suggest new studies to be conducted with this characteristic. The low number of papers included with this theme is also because only DL was tested, or the average for the 2 legs was used as a result. Therefore, the evaluation of both legs and the exposure of these results to the scientific community is also extremely important.

Another point that may influence the results is the lack of information on the young players' training routine. It is suggested that future studies describe this in more detail, as well as indicate the playing positions of the sample evaluated, as they can also impact on the results [38]. A need for prospective studies highlighting mechanisms that might increase injury risk in young players was also verified.

As practical applications concerning isokinetic evaluation, we suggest using the proposed protocol and the reference values for the conventional and functional $\mathrm{H} / \mathrm{Q}$ ratio at $60 \% \mathrm{~s}$ and $180 \%$, as well as for BD. For flexibility, we suggest increased use of goniometry to evaluate hip flexion movement and the application of reference values for angulation and BD. Moreover, it becomes essential to monitor these variables and their possible interactions throughout young soccer athletes' formation process. This will allow to evaluate and plan training strategies to prevent and reduce potential muscle imbalances. 


\section{Conclusions}

In young players, soccer does not generate asymmetries of strength, and the H/Q ratio between limbs does not change the expected values for the H/Q ratio and does not significantly generate BD. For flexibility, it is easier to create asymmetries between limbs, with DL more flexible than NDL; however, more studies are needed to corroborate this statement. The monitoring of these characteristics and the use of interventions to prevent these asymmetries and/or imbalances or to restore symmetrical patterns have become essential since the beginning of the individuals' sports lives. Imbalances for the H/Q ratio, asymmetries, and $\mathrm{BD}$ for strength and flexibility are not natural aspects of the sport and should be avoided in young soccer players. These individuals are expected to remain within the suggested standards. While emphasizing that flexibility may be easier to be asymmetrical, and because of that, it deserves greater attention within the training routine.

As recommended for further studies we enumerate using the suggested protocol for isokinetic strength evaluation, utilizing a goniometer to measure flexibility, making comparisons for asymmetries and imbalances in H/Q and BD (with the cut-offs/normative values suggested), and, in addition, trying to establish a relationship between these characteristics and an increased injury risk in young soccer players.

\section{Acknowledgements}

The authors would like to thank all the researchers who were contacted during the preparation of the study and answered the questions promptly.

\section{Disclosure statement}

No author has any financial interest or received any financial benefit from this research.

\section{Conflict of interest}

The authors state no conflict of interest.

\section{References}

1. FIFA. Youth football. Zurich: FIFA; 2016.

2. Daneshjoo A, Rahnama N, Mokhtar AH, Yusof A. Bilateral and unilateral asymmetries of isokinetic strength and flexibility in male young professional soccer players. J Hum Kinet. 2013;36:45-53; doi: 10.2478/hukin2013-0005.

3. Akbulut T, Agopyan A. Effects of an eight-week proprioceptive neuromuscular facilitation stretching program on kicking speed and range of motion in young male soccer players. J Strength Cond Res. 2015;29(12): 3412-3423; doi: 10.1519/JSC.0000000000001015.
4. DeLang MD, Kondratek M, DiPace LJ, Hew-Butler T. Collegiate male soccer players exhibit between-limb symmetry in body composition, muscle strength, and range of motion. Int J Sports Phys Ther. 2017;12(7): 1087-1094; doi: 10.16603/ijspt20171087.

5. Fousekis K, Tsepis E, Poulmedis P, Athanasopoulos S, Vagenas G. Intrinsic risk factors of non-contact quadriceps and hamstring strains in soccer: a prospective study of 100 professional players. Br J Sports Med. 2011; 45(9):709-714; doi: 10.1136/bjsm.2010.077560.

6. Terreri ASAP, Greve JMD, Amatuzzi MM. Isokinetic evaluation of athletes' knee [in Portuguese]. Rev Bras Med Esporte. 2001;7(5):170-174; doi: 10.1590/S151786922001000200004.

7. Costa Silva JRL, Detanico D, Dal Pupo J, de la Rocha Freitas C. Bilateral asymmetry of knee and ankle isokinetic torque in soccer players u20 category. Rev Bras Cineantropom Desempenho Hum. 2015;17(2):195-204; doi: 10.5007/1980-0037.2015v17n2p195.

8. De Lira CAB, Mascarin NC, Vargas VZ, Vancini RL, Andrade MS. Isokinetic knee muscle strength profile in Brazilian male soccer, futsal, and beach soccer players: a cross-sectional study. Int J Sports Phys Ther. 2017;12(7):1103-1110; doi: 10.16603/ijspt20171103.

9. Śliwowski R, Jadczak Ł, Hejna R, Wieczorek A. The effects of individualized resistance strength programs on knee muscular imbalances in junior elite soccer players. PLoS One. 2015;10(12):e0144021; doi: 10.1371/journal.pone.0144021.

10. Maly T, Zahalka F, Mala L. Unilateral and ipsilateral strength asymmetries in elite youth soccer players with respect to muscle group and limb dominance. Int $\mathrm{J}$ Morphol. 2016;34(4):1339-1344; doi: 10.4067/S071795022016000400027.

11. Gür H, Akova B, Pündük Z, Küçükoğlu S. Effects of age on the reciprocal peak torque ratios during knee muscle contractions in elite soccer players. Scand J Med Sci Sports. 1999;9(2):81-87; doi: 10.1111/j.1600-0838.1999. tb00213.x.

12. Seabra A, Marques E, Brito J, Krustrup P, Abreu S, Oliveira J, et al. Muscle strength and soccer practice as major determinants of bone mineral density in adolescents. Joint Bone Spine. 2012;79(4):403-408; doi: 10.1016/j.jbspin.2011.09.003.

13. Daneshjoo A, Mokhtar A, Rahnama N, Yusof A. The effects of injury prevention warm-up programmes on knee strength in male soccer players. Biol Sport. 2013; 30(4):281-288; doi: 10.5604/20831862.1077554.

14. Rey E, Padrón-Cabo A, Barcala-Furelos R, Mecías-Calvo M. Effect of high and low flexibility levels on physical fitness and neuromuscular properties in professional soccer players. Int J Sports Med. 2016;37(11):878-883; doi: 10.1055/s-0042-109268.

15. Sanz A, Pablos C, Ballester R, Sánchez-Alarcos JV, Huertas F. Range of motion and injury occurrence in elite Spanish soccer academies. Not only a hamstring shortening-related problem. J Strength Cond Res. 2020; 
34(7):1924-1932; doi: 10.1519/JSC.000000000000 3302.

16. Ramos S, Simão R, Herdy C, Costa P, Dias I. Relationship between strength and flexibility levels in young soccer players. Apunts Sports Med. 2019;54(203):85-90; doi: 10.1016/j.apunts.2018.11.005.

17. Pfirrmann D, Herbst M, Ingelfinger P, Simon P, Tug S. Analysis of injury incidences in male professional adult and elite youth soccer players: a systematic review. J Athl Train. 2016;51(5):410-424; doi: 10.4085/10626050-51.6.03.

18. McCall A, Carling C, Davison M, Nedelec M, Le Gall F, Berthoin S, et al. Injury risk factors, screening tests and preventative strategies: a systematic review of the evidence that underpins the perceptions and practices of 44 football (soccer) teams from various premier leagues. Br J Sports Med. 2015;49(9):583-589; doi: 10.1136/bjsports-2014-094104.

19. Moher D, Liberati A, Tetzlaff J, Altman DG. Preferred Reporting Items for Systematic Reviews and Meta-Analyses: the PRISMA statement. PLoS Med. 2009;6(7); e1000097; doi: 10.1371/journal.pmed.1000097.

20. Downs SH, Black N. The feasibility of creating a checklist for the assessment of the methodological quality both of randomised and non-randomised studies of health care interventions. J Epidemiol Community Health. 1998;52(6):377-384; doi: 10.1136/jech.52.6.377.

21. Figueiredo T, de Salles BF, Dias I, Reis VM, Fleck SJ, Simão R. Acute hypotensive effects after a strength training session: a review. Int Sportmed J. 2014;15(3): 308-329.

22. Forbes H, Sutcliffe S, Lovell A, McNaughton LR, Siegler JC. Isokinetic thigh muscle ratios in youth football: effect of age and dominance. Int J Sports Med. 2009; 30(8):602-606; doi: 10.1055/s-0029-1202337.

23. Lehance C, Binet J, Bury T, Croisier JL. Muscular strength, functional performances and injury risk in professional and junior elite soccer players. Scand J Med Sci Sports. 2009;19(2):243-251; doi: 10.1111/ j.1600-0838.2008.00780.x.

24. Daneshjoo A, Mokhtar AH, Rahnama N, Yusof A. The effects of injury preventive warm-up programs on knee strength ratio in young male professional soccer players. PLoS One. 2012;7(12):e50979; doi: 10.1371/journal.pone.0050979.

25. Lehnert M, Xaverová Z, De Ste Croix M. Changes in muscle strength in U19 soccer players during an annual training cycle. J Hum Kinet. 2014;42(1):175-185; doi: 10.2478/hukin-2014-0072.

26. Chiamonti Bona C, Tourinho Filho H, Izquierdo M, Pires Ferraz RM, Marques MC. Peak torque and muscle balance in the knees of young U-15 and U-17 soccer athletes playing various tactical positions. J Sports Med Phys Fitness. 2017;57(7-8):923-929; doi: 10.23736/ S0022-4707.16.06458-6.

27. Eustace SJ, Page RM, Greig M. Angle-specific isokinetic metrics highlight strength training needs of elite youth
soccerplayers.JStrength CondRes. 2020;34(11):3258 3265; doi: 10.1519/JSC.0000000000002612.

28. Harper DJ, Jordan AR, Kiely J. Relationships between eccentric and concentric knee strength capacities and maximal linear deceleration ability in male academy soccer players. J Strength Cond Res. 2021;35(2):465472; doi: 10.1519/JSC.0000000000002739.

29. Croisier J-L, Ganteaume S, Binet J, Genty M, Ferret J-M. Strength imbalances and prevention of hamstring injury in professional soccer players: a prospective study. Am J Sports Med. 2008;36(8):1469-1475; doi: 10.1177/ 0363546508316764.

30. DeLang MD, Rouissi M, Bragazzi NL, Chamari K, Salamh PA. Soccer footedness and between-limbs muscle strength: systematic review and meta-analysis. Int J Sports Physiol Perform. 2019;14(5):551-562; doi: 10.1123/ijspp.2018-0336.

31. Manfredini Baroni B, Ruas CV, Ribeiro-Alvares JB, Silveira Pinto R. Hamstring-to-quadriceps torque ratios of professional male soccer players: a systematic review. J Strength Cond Res. 2020;34(1):281-293; doi: 10.1519/JSC.0000000000002609.

32. Read PJ, Jimenez P, Oliver JL, Lloyd RS. Injury prevention in male youth soccer: current practices and perceptions of practitioners working at elite English academies. J Sports Sci. 2018;36(12):1423-1431; doi: 10.1080/ 02640414.2017.1389515.

33. Otero-Esquina C, de Hoyo Lora M, Gonzalo-Skok Ó, Domínguez-Cobo S, Sánchez H. Is strength-training frequency a key factor to develop performance adaptations in young elite soccer players? Eur J Sport Sci. 2017;17(10):1241-1251; doi: 10.1080/17461391.2017. 1378372.

34. Sporis G, Vucetic V, Jovanovic M, Jukic I, Omrcen D. Reliability and factorial validity of flexibility tests for team sports. J Strength Cond Res. 2011;25(4):11681176; doi: 10.1519/JSC.0b013e3181cc2334.

35. Witvrouw E, Danneels L, Asselman P, D'Have T, Cambier D. Muscle flexibility as a risk factor for developing muscle injuries in male professional soccer players: a prospective study. Am J Sports Med. 2003;31(1):4146; doi: 10.1177/03635465030310011801.

36. Ribeiro-Alvares JB, Pinto Dornelles M, Gassen Fritsch C, de Lima-e-Silva FX, Menezes Medeiros T, Severo-Silveira $L$, et al. Prevalence of hamstring strain injury risk factors in professional and under-20 male football (soccer) players. J Sport Rehabil. 2020;29(3):339-345; doi: 10.1123/jsr.2018-0084.

37. Vasconcellos F, Salles P, Achour A, de Mello DB, Dantas EHM. The vertical jump height of soccer players after static overstretching. Hum Mov. 2012;13(1):4-7; doi: 10.2478/v10038-011-0047-1.

38. Herdy CV, Galvao P, Costa e Silva G, Ramos S, Simao R, Pedrinelli A, et al. Knee flexion and extension strength in young Brazilian soccer players: the effect of age and position. Hum Mov. 2018;19(3):23-29; doi: 10.5114/ hm.2018.76076. 